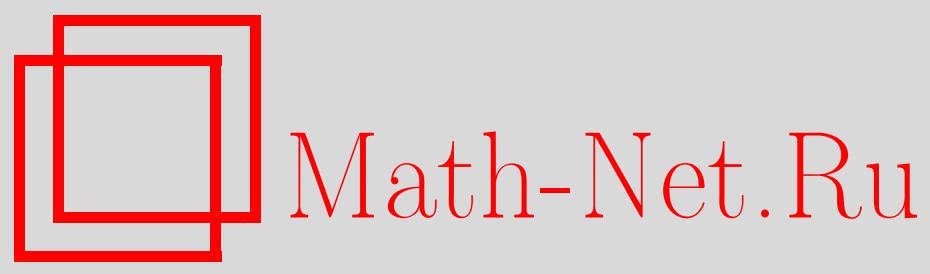

Г. И. Архипов, С. Б. Гашков, М. П. Минеев, С. М. Никольский, В. А. Садовничий, О математических работах Владимира Николаевича Чубарикова, Дискрет. матем., 2012, том 24, выпуск 2, 4-20

DOI: https://doi.org/10.4213/dm1180

Использование Общероссийского математического портала Math-Net.Ru подразумевает, что вы прочитали и согласны с пользовательским соглашением http://www . mathnet.ru/rus/agreement

Параметры загрузки:

IP : 54.162 .27 .143

26 апреля 2023 г., 18:10:17 
УДК 519.1

\title{
О математических работах Владимира Николаевича Чубарикова
}

\author{
() 2012 г. Г. И. Архипов, С. Б. Гашков, М. П. Минеев, \\ С. М Никольский, В. А. Садовничий
}

5 сентября 2011 г. исполнилось шестьдесят лет известному российскому математику, доктору физико-математических наук, профессору Владимиру Николаевичу Чубарикову. Последние пять лет, по приказу ректора Московского государственного университета имени М. В. Ломоносова В. А. Садовничего, он возглавляет механико-математический факультет МГУ.

В 1959 г. Володя Чубариков пошел учиться в сельскую школу. В 1960 г. его вместе со старшим братом Анатолием направили учиться в Михайловскую школу-интернат Волгоградской области. Следует сказать, что к этому моменту в семье Чубариковых было восемь детей, - четыре сестры и четыре брата. В Михайловской школе-интернате был собран хороший педагогический коллектив из молодых учителей. В частности, учителем математики был будущий отличник народного просвещения Владимир Сергеевич Попов, который оказал заметное влияние на математическое развитие Володи Чубарикова. Начиная с шестого класса, Володя неизменно побеждал на областных математических олимпиадах в Волгоградской области. Вместе с тем он отлично успевал и по другим предметам. В областных газетах публиковались его заметки и стихи. Он всерьез думал о журналистской деятельности, но математика взяла свое. Единственный раз в девятом классе он был включен в состав команды Волгоградской области на Всесоюзную математическую олимпиаду в г. Ленинграде. Там он успешно прошел отбор в колмогоровский интернат при МГУ и десятый класс заканчивал в Москве.

В 1969 г. В. Н. Чубариков окончил специализированную школу-интернат №18 физико-математического профиля при Московском государственном университете имени М. В. Ломоносова и поступил на механико-математический факультет, с которым, как оказалось впоследствии, неразрывно связана его дальнейшая научная и педагогическая деятельность. Учился В. Н. Чубариков легко и с удовольствием. По математическим дисциплинам он получал только отличные оценки. Уже на первом курсе началась его научная деятельность. Он нашел новую границу нулей для $L$-функций Дирихле, отвечающих модулям специального вида, и существенно улучшил остаток в асимптотической формуле для количества простых чисел в арифметической прогрессии с соответствующей разностью.

В 1974 г. В. Н. Чубариков закончил механико-математический факультет МГУ и поступил в аспирантуру отделения математики того же факультета. Его многолетний сосед по общежитию Олег Вячеславович Вьюгин, ныне известный в России специалист в области финансов, привлек в аспирантские годы Владимира Николаевича к комсомольской деятельности. Здесь у него обнаружились хорошие организаторские способности; помноженные на крестьянское трудолюбие и добросовестное отношение к делу, они привели 
его в 1977 г. к посту первого секретаря комитета ВЛКСМ механико-математического факультета МГУ. В 1979 г. общественная деятельность Владимира Николаевича временно прервалась. Но когда встал вопрос об оставлении на преподавательскую работу на механико-математическом факультете МГУ, обнаружилось. что у В. Н. Чубарикова к тому времени было более десятка печатных научных работ, он своевременно в 1977 г. защитил кандидатскую диссертацию на тему «Кратные тригонометрические суммы» (научный руководитель - профессор А. А. Карацуба). Кроме того, он решил проблему Хуа Ло-кена о показателях сходимости особого интеграла и особого ряда в проблеме Терри. При его участии была разработана теория кратных тригонометрических сумм, аналогичная теории сумм Г. Вейля, построенная ранее И. М. Виноградовым, и которая в 1977 г. была признана лучшей математической работой в масштабе Академии наук СССР за последние 5 лет. По результатам этих исследований была подготовлена к печати монография «Кратные тригонометрические суммы», изданная в 1980 г. в Трудах Математического института имени В. А. Стеклова АН СССР. Академик И. М. Виноградов обратил особое внимание на исследования В. Н. Чубарикова по оценкам тригонометрических интегралов и оказал ему определенную научную поддержку. По его рекомендации в 1980 г. В. Н. Чубариков был назначен ученым секретарем специализированного докторского совета по защите диссертаций на механико-математическом факультете МГУ, а в 1982 г. он был назначен заместителем декана факультета. В этой должности он проработал до безвременной кончины декана механико-математического факультета МГУ академика О. Б. Лупанова в мае 2006 г. С тех пор В. Н. Чубариков возглавляет механико-математический факультет МГУ.

Математические исследования В. Н. Чубарикова относятся к аналитической теории чисел, математическому анализу и дискретной математике. Как было отмечено, начало его математической деятельности связано с аналитической теорией чисел, которая до сих пор является основой его научных устремлений. Исследования В. Н. Чубарикова во многом продолжают исследования И. М. Виноградова по фундаментальным проблемам теории чисел, которые восходят к работам основоположников отечественной математики Л. Эйлеру и П. Л. Чебышёву. В своем докладе «Простые числа, дзета-функция Римана и тригонометрические суммы» на общеинститутском семинаре 16 июня 2011 г. в Математическом институте имени В. А. Стеклова Российской Академии наук В. Н. Чубариков четко подчеркнул эту преемственность. В работе «Об одном преобразовании числовых рядов» (1879) П. Л. Чебышёв нашел, что тождество Эйлера

$$
\zeta(s)=\prod_{p}\left(1-p^{-s}\right)^{-1}
$$

и формула Чебышёва, заменяющая сумму логарифмов натуральных чисел (до известного предела) суммами, относящимися к простым числам:

$$
\sum_{n \leqslant x} \log n=\sum_{m \leqslant x} \psi(x / m),
$$

являются следствием одного общего тождества

$$
\sum_{n=2}^{\infty} f(n) \log n=\sum_{p} F(p) \log p,
$$

где

$$
F(t)=\sum_{n=1}^{\infty} \sum_{m=1}^{\infty} f\left(n t^{m}\right) .
$$


Из последней формулы тождество Эйлера получается при $f(t)=t^{-s}$, а тождество Чебышёва - при

$$
f(n)= \begin{cases}1, & n \leqslant x \\ 0, & n>x\end{cases}
$$

Подобное тождество лежит в основе метода И. М. Виноградова оценок тригонометрических сумм с простыми числами:

$$
\Phi(1)+\sum_{H<y \leqslant N} \Phi(y)=\sum_{d m \leqslant N} \sum_{m} \mu(d) \Phi(d m),
$$

где $H$ - любое число с условием $1<H \leqslant \sqrt{N}, y$ пробегает числа, не делящиеся на простые числа, не превосходящие $H, d$ пробегает произведение простых чисел (включая пустое произведение, равное 1$)$, не превосходящих $H$, наконец, $m$ пробегает натуральные числа. Тождество Эйлера получается отсюда при $\Phi(n)=n^{-s}$ путем предельного перехода.

В 1937 г. И.М. Виноградов нашел нетривиальную оценку для суммы $\sum_{p \leqslant N} e^{2 \pi i \alpha p}$, с помощью которой вывел асимптотическую формулу для числа представлений нечетного числа суммой трех нечетных простых чисел (тернарная проблема Гольдбаха). В том же 1937 г. он получил нетривиальную оценку для тригонометрической суммы $\sum_{p \leqslant N} e^{2 \pi i\left(\alpha_{n} p^{n}+\ldots+\alpha_{1} p\right)}$, где суммирование ведется по простым числам $p$. Отметим, что идея И. М. Виноградова оценки сумм с простыми числами состояла в том, что он с помощью указанного выше тождества сводил эти суммы к небольшому количеству кратных сумм специального вида, переменные суммирования в которых были независимы. Для кратных же сумм несколько ранее (1934 г.) И. М. Виноградов открыл способ получения их нетривиальных оценок. В частности, это позволило ему получить выдающееся продвижение в проблеме Варинга о представлении натуральных чисел суммами степеней натуральных чисел. И. М. Виноградов сразу оценил широкие перспективы своего метода. С данного момента он стал говорить о создании нового метода тригонометрических сумм в теории чисел, хотя основы метода заложены им за 20 лет до этого. Отметим также, что новый метод выдвинул ряд новых задач в теории чисел и анализе. И. М. Виноградов в монографии «Метод тригонометрических сумм в теории чисел» (1947 г.) выделил три актуальных направления исследований, связанных с его методом:

- оценки кратных тригонометрических сумм с вещественной функцией в экспоненте,

- распределение значений арифметических функций от многих переменных,

- диофантов анализ для целозначных функций от большого числа переменных, причем переменные могут пробегать различные множества значений, например, множество простых чисел.

Перейдем теперь к более полному описанию научной работы В. Н. Чубарикова.

\section{1. Кратные тригонометрические суммы Г. Вейля с простыми числами}

Венцом метода И. М. Виноградова в применении к оценкам однократных тригонометрических сумм Г. Вейля было получение оценок тригонометрических сумм по простым 
числам приблизительно такой же силы, как и для сумм по сплошному промежутку суммирования. Для кратных сумм подобная задача ставилась самим Виноградовым. Во второй половине 70-х годов над ней активно работал А. А. Карацуба.

В 1984 г. В. Н. Чубариков решил эту проблему И. М. Виноградова. Он нашел нетривиальную оценку для кратной тригонометрической сумме по простым числам с многочленом общего вида в экспоненте $\sum_{p_{1} \leqslant N_{1}} \cdots \sum_{p_{r} \leqslant N_{r}} e^{2 \pi i F\left(p_{1}, \ldots, p_{r}\right)}$, где переменные $p_{1}, \ldots, p_{r}$ пробегают все последовательные простые числа и

$$
F\left(x_{1}, \ldots, x_{r}\right)=\sum_{t_{1}=0}^{n_{1}} \ldots \sum_{t_{r}=0}^{n_{r}} \alpha\left(t_{1}, \ldots, t_{r}\right) x_{1}^{t_{1}} \ldots x_{r}^{t_{r}}, \quad \alpha(0, \ldots, 0)=0 .
$$

Эта оценка близка к той, которую Г. И. Архипов и В. Н. Чубариков получили для кратных тригонометрических сумм Г. Вейля по сплошным промежуткам суммирования.

\section{2. Моменты тригонометрических сумм Г. Вейля}

К концу шестидесятых годов двадцатого столетия сложилась парадоксальная ситуация. Для однократных сумм Г. Вейля И. М. Виноградов разработал теорию, основу которой составила точная оценка моментов этих сумм. Она получила название теоремы И. М. Виноградова о среднем.

Пусть $n \geqslant 2$ - натуральное число, $\alpha_{1}, \ldots, \alpha_{n}-$ вещуественные числа. Тогда для интеграла $J$ вида

$$
J=J(P ; k, n)=\int_{0}^{1} \ldots \int_{0}^{1}\left|\sum_{x \leqslant P} e^{2 \pi i\left(\alpha x^{n}+\ldots+\alpha_{1} x\right)}\right|^{2 k} d \alpha_{n} \ldots d \alpha_{1}
$$

справедлива оиченка

$$
J=J(P ; k, n) \leqslant D P^{2 k-\frac{1}{2} n(n+1)+\delta(\tau)},
$$

где

$$
\begin{aligned}
\delta & =\frac{1}{2} n(n+1)(1-1 / n)^{\tau}, \\
D & =D(\tau)=(n \tau)^{6 n \tau}(2 n)^{4 n(n+1) \tau} .
\end{aligned}
$$

В то же время для кратных сумм Г. Вейля ничего не было известно, хотя сама задача была поставлена Виноградовым еще в сороковые годы. Первые оценки кратных сумм были получены Г. И. Архиповым в 1971 г. и опубликованы им в 1974 г. В 1975 г. Г. И. Архипов и В. Н. Чубариков решили проблему моментов для кратных сумм, на основе которой была построена теория, подобная теории И. М. Виноградова для однократных сумм Г. Вейля. Другими словами, они получили правильную оценку сверху по порядку растущих параметров $\bar{P}=\left(P_{1}, \ldots, P_{r}\right), P_{1}=\min \left\{P_{1}, \ldots, P_{r}\right\}$ при $P_{1} \rightarrow \infty$ следующей величины:

$$
J=J(\bar{P} ; \bar{n}, k)=\int \cdots \int_{\Omega}|S(\Omega)|^{2 k} d \Omega
$$


где $S(\Omega)$ - кратная тригонометрическая сумма Г. Вейля вида

$$
S(\Omega)=\sum_{x_{1} \leqslant P_{1}} \cdots \sum_{x_{r} \leqslant P_{r}} \exp \left\{2 \pi i F\left(x_{1}, \ldots, x_{r}\right)\right\}
$$

И

$$
F\left(x_{1}, \ldots, x_{r}\right)=\sum_{t_{1}=0}^{n_{1}} \ldots \sum_{t_{r}=0}^{n_{r}} \alpha\left(t_{1}, \ldots, t_{r}\right) x_{1}^{t_{1}} \ldots x_{r}^{t_{r}}, \quad \alpha(0, \ldots, 0)=0,
$$

$\Omega$ - набор вещественных коэффициентов $\alpha(\bar{t})=\alpha\left(t_{1}, \ldots, t_{r}\right)$ многочлена $F\left(x_{1}, \ldots, x_{r}\right)$, $n_{1}, \ldots n_{r} \geqslant 1, \bar{t}=\left(t_{1}, \ldots, t_{r}\right)$ и

$$
d \Omega=\prod_{t_{1}=0}^{n_{1}} \ldots \prod_{\substack{t_{r}=0 \\ t_{1}+\ldots+t_{r} \geqslant 1}}^{n_{r}} d \alpha\left(t_{1}, \ldots, t_{r}\right) .
$$

\section{3. Тригонометрические интегралы}

В рамках теории тригонометрических сумм исследовались тригонометрические интегралы. Приведем последний вариант оценки И. М. Виноградова (1981 г.).

Пусть $\alpha=\max \left\{\left|\alpha_{n}\right|, \ldots,\left|\alpha_{1}\right|\right\}$. Тогда

$$
\left|\int_{0}^{1} e^{2 \pi i\left(\alpha_{n} x^{n}+\ldots+\alpha_{1} x\right)} d x\right| \leqslant \min \left\{1,32 \alpha^{-1 / n}\right\} .
$$

В 1976 г. В. Н. Чубариков получил следующую оценку кратного тригонометрического интеграла.

Пусть $F\left(x_{1}, \ldots, x_{r}\right)$ - многочлен с вещественными коэффициентами $\alpha(\bar{t}), n=$ $\max \left\{n_{1}, \ldots, n_{r}\right\}, \alpha=\max _{\bar{t}}|\alpha(\bar{t})|$. Тогда

$$
\left|\int_{0}^{1} \ldots \int_{0}^{1} e^{2 \pi i F\left(x_{1}, \ldots, x_{r}\right)} d x_{1} \ldots d x_{r}\right| \leqslant \min \left\{1,32 \alpha^{-1 / n} \ln ^{r-1}(\alpha+2)\right\} .
$$

Отдельной проблемой математики является определение показателя сходимости $\gamma$ для моментов тригонометрических интегралов, т.е. нахождение такого вещественного числа $\gamma$, что при $2 k>\gamma$ сходится интеграл

$$
\theta(k)=\int_{-\infty}^{\infty} \ldots \int_{-\infty}^{\infty}\left|\int_{0}^{1} e^{2 \pi i\left(\alpha_{n} x^{n}+\ldots+\alpha_{1} x\right)} d x\right|^{2 k} d \alpha_{n} \ldots d \alpha_{1},
$$

а при $2 k \leqslant \gamma$ он расходится. Эта проблема была сформулирована Хуа Ло-кеном в 1952 г. Он получил первые оценки сверху величины $\gamma$. В 1978 г. Г. И. Архипов, А. А. Карацуба и В. Н. Чубариков решили проблему Хуа Ло-кена. Было установлено, что интеграл $\theta(k)$ сходится при $2 k>\frac{1}{2} n(n+1)+1$ и расходится при $2 k \leqslant \frac{1}{2} n(n+1)+1$.

Для “выщербленного” многочлена

$$
f(x)=\alpha_{n} x^{n}+\ldots+\alpha_{m} x^{m}+\alpha_{r} x^{r},
$$


где $n>\ldots>m>r, n+\ldots+m+r<\frac{1}{2} n(n+1)$, был обнаружен следующий неожиданный эффект.

Показатель сходимости $\gamma_{0}$ интеграла

$$
\theta_{0}(k)=\int_{-\infty}^{\infty} \ldots \int_{-\infty}^{\infty}\left|\int_{0}^{1} e^{2 \pi i f(x)} d x\right|^{2 k} d \alpha_{n} \ldots d \alpha_{m} d \alpha_{r}
$$

равен $\gamma_{0}=n+\ldots+m+r$.

Значения показателя сходимости для кратных тригонометрических интегралов на сегодняшний день не найдены.

\section{4. Полные рациональные тригонометрические суммы}

Хуа Ло-кен получил точную по порядку роста величины $q$ оценку модуля полной рациональной тригонометрической суммы

$$
S=S(q, f)=\sum_{x=1}^{q} e^{2 \pi i f(x) / q}, \quad|S| \leqslant e^{n A(n)} q^{1-1 / n},
$$

где последние значения функций $A(n)$ были найдены Чэном Джун-раном:

$$
\begin{aligned}
& A(3)=6,1, \quad A(4)=5,5, \quad A(5)=5, \quad A(6)=4,7, \quad A(7)=4,4 \text {, } \\
& A(8)=4,2, \quad A(9)=4,05, \quad A(n)=4, \quad n \geqslant 10 \text {, }
\end{aligned}
$$

И

$$
f(x)=a_{n} x^{n}+\ldots+a_{1} x
$$

- многочлен с целыми коэффициентами, $\left(a_{n}, \ldots, a_{1}, q\right)=1, q \geqslant 1, n \geqslant 3$.

\section{1. Кратные рациональные тригонометрические суммы}

В. Н. Чубариков получил следующую верхнюю границу для полной рациональной кратной тригонометрической суммы.

Пусть $n \geqslant 2-$ целое число, $n=\max \left\{n_{1}, \ldots, n_{r}\right\}, q-$ натуральное число, и пусть

$$
S(q, F)=\sum_{x_{1}=1}^{q} \ldots \sum_{x_{r}=1}^{q} e^{2 \pi i F\left(x_{1}, \ldots, x_{r}\right) / q},
$$

где

$$
F\left(x_{1}, \ldots, x_{r}\right)=\sum_{t_{1}=0}^{n_{1}} \ldots \sum_{t_{r}=0}^{n_{r}} a\left(t_{1}, \ldots, t_{r}\right) x_{1}^{t_{1}} \cdots x_{r}^{t_{r}}
$$

- многочлен с цуелыми коэффициентами, в совокупности взаимно простыли с q. Тогда

$$
|S(q, F)| \leqslant e^{7 n r} 3^{r \omega(q)}(\tau(q))^{r-1} q^{r-1 / n} .
$$




\section{2. Показатель сходимости особого ряда в проблеме Терри}

В 1952 г. Хуа Ло-кен решил задачу о показателе сходимости среднего значения полной рациональной тригонометрической суммы.

Пусть $n \geqslant 3$,

$$
f(x)=\frac{a_{1}}{q_{1}} x+\ldots+\frac{a_{n}}{q_{n}} x^{n},
$$

$\left(a_{1}, q_{1}\right)=\ldots=\left(a_{n}, q_{n}\right)=1$, и $q=q_{1} \cdots q_{n}$. Среднее значение $\sigma$ полной рациональной тригонометрической суммы

$$
S(q, f)=\sum_{x=1}^{q} e^{2 \pi i f(x)}
$$

(особый ряд в проблеме Терри) определяется выражением

$$
\sigma=\sum_{q_{n}=1}^{\infty} \ldots \sum_{q_{1}=1}^{\infty} \sum_{a_{n}=0}^{q_{n}-1} \ldots \sum_{a_{1}=0}^{q_{1}-1}\left|q^{-1} S(q, f)\right|^{2 k}
$$

где штрих в суммировании означает, что $a_{s}$ пробегает приведенную систему вычетов по модулю $q_{s}, s=1, \ldots, n$.

Хуа Ло-кен доказал следующее утверждение.

Особый ряд б сходится при $2 k>\frac{1}{2} n(n+1)+2$ и расходится при $2 k \leqslant \frac{1}{2} n(n+1)+2$.

Пусть, далее, $1 \leqslant m<r<\ldots \leqslant n-$ натуральные числа, и пусть $n \geqslant 3$,

$$
f(x)=\frac{a_{m}}{q_{m}} x^{m}+\ldots+\frac{a_{n}}{q_{n}} x^{n},
$$

$\left(a_{m}, q_{m}\right)=\ldots=\left(a_{n}, q_{n}\right)=1$, и $q=q_{m} \cdots q_{n}$. Определим среднее значение полной рациональной тригонометрической суммы с “выщербленным” многочленом в виде

$$
\sigma_{0}=\sum_{q_{n}=1}^{\infty} \ldots \sum_{q_{m}=1}^{\infty} \sum_{a_{n}=0}^{q_{n}-1} \ldots \sum_{a_{m}=0}^{q_{m}-1}\left|q^{-1} S(q, f)\right|^{2 k}
$$

В 1981 г. В.Н. Чубариков нашел показатель сходимости особого ряда для “выщербленного" многочлена.

Особый ряд $\sigma_{0}$ для $1 \leqslant m<r<\ldots<n, m+r+\ldots+n<\frac{1}{2} n(n+1)$ сходится при $2 k>m+r+\ldots+n+1$ и расходится при $2 k \leqslant m+r+\ldots+n+1$.

\section{3. Показатель сходимости особого ряда в многомерной проблеме Терри}

Пусть $n \geqslant 2, k \geqslant 1, r \geqslant 1-$ натуральные числа, $a\left(t_{1}, \ldots, t_{r}\right)$ и $q\left(t_{1}, \ldots, t_{r}\right)-$ наборы натуральных чисел, где $0 \leqslant t_{1}, \ldots, t_{r} \leqslant n$, и

$$
q=\prod_{0 \leqslant t_{1}, \ldots, t_{r} \leqslant n} q\left(t_{1}, \ldots, t_{r}\right), q(0, \ldots, 0)=1, \quad a(0, \ldots, 0)=0 .
$$


Тогда особый ряд в многомерной проблеме Терри имеет вид

$$
\sigma_{r}=\sum_{q(n, \ldots, n)=1}^{\infty} \ldots \sum_{q(0, \ldots, 1)=1}^{\infty} \sum_{a(n, \ldots, n)=1}^{q(n, \ldots, n)} \ldots \sum_{a(0, \ldots, 1)=1}^{q(0, \ldots, 1)}\left|q^{-r} \sum_{x_{1}=1}^{q} \ldots \sum_{x_{r}=1}^{q} e^{2 \pi i F\left(x_{1}, \ldots, x_{r}\right)}\right|^{2 k},
$$

где

$$
F\left(x_{1}, \ldots, x_{r}\right)=\sum_{t_{1}=0}^{n} \ldots \sum_{t_{r}=0}^{n} \frac{a\left(t_{1}, \ldots, t_{r}\right)}{q\left(t_{1}, \ldots, t_{r}\right)} x_{1}^{t_{1}} \cdots x_{r}^{t_{r}}
$$

- многочлен с рациональными коэффициентами, а штрих при знаке суммирования означает, что $\left(a\left(t_{1}, \ldots, t_{r}\right), q\left(t_{1}, \ldots, t_{r}\right)\right)=1$.

Из оценки суммы в $\$ 4.1$ следует, что $\sigma_{r}$ сходится при $2 k>n(n+1)^{r}$. В. Н. Чубариков в 2006 г. доказал, что ряд $\sigma_{r}$ расходится при $2 k r \leqslant r T+1$, где

$$
\begin{aligned}
T & =\sum_{t_{1}=0}^{n} \ldots \sum_{t_{r}=0}^{n} \min \left\{t_{1}, \ldots, t_{r}\right\} \\
& =\frac{1}{r+1} \sum_{s=1}^{r+1}\left(\begin{array}{c}
r+1 \\
s
\end{array}\right) B_{r+1-s}(n+1)^{s} \sim \frac{(n+1)^{r+1}}{r+1},
\end{aligned}
$$

где $B_{s}, s \geqslant 0$ - числа Бернулли.

\section{5. Аддитивные задачи варинговского типа с простыми числами}

Исследования по тригонометрическим суммам привели В. Н. Чубарикова к рассмотрению проблемы Гильберта-Камке в простых числах, т.е. к проблеме разрешимости в простых числах $p_{1}, \ldots, p_{k}$ системы уравнений

$$
\begin{gathered}
p_{1}+\ldots+p_{k}=N_{1}, \\
\ldots \\
p_{1}^{n}+\ldots+p_{k}^{n}=N_{n},
\end{gathered}
$$

где $N_{1}, \ldots, N_{n}-$ наборы натуральных чисел, удовлетворяющие условиям

$$
N_{k}=P^{k}\left(\gamma_{k}+o(1)\right), \quad \gamma_{k} \neq 0, \quad k=1, \ldots, n,
$$

и $P$ - некоторый вещественный параметр, стремящийся к бесконечности.

Эту проблему условно и независимо друг от друга решили К. К. Марджанишвили и Хуа Ло-кен. Полное ее решение дал В. Н. Чубариков в 1985 г. Более того, в 1985 г. В. Н. Чубариковым была решена общая многомерная аддитивная проблема следующего вида:

$$
p_{11}^{t_{1}} \cdots p_{r 1}^{t_{r}}+\ldots+p_{1 k}^{t_{1}} \cdots p_{r k}^{t_{r}}=N\left(t_{1}, \ldots, t_{r}\right),
$$

где $0 \leqslant t_{1} \leqslant n_{1}, \ldots, 0 \leqslant t_{r} \leqslant n_{r}, t_{1}+\ldots+t_{r} \geqslant 1$, причем наборы $N\left(t_{1}, \ldots, t_{r}\right)$ удовлетворяют условиям регулярности

$$
N\left(t_{1}, \ldots, t_{r}\right)=P_{1}^{t_{1}} \cdots P_{r}^{t_{r}}\left(\gamma\left(t_{1}, \ldots, t_{r}\right)+o(1)\right),
$$


и $\gamma\left(t_{1}, \ldots, t_{r}\right) \neq 0$ при $P_{1}=\min \left\{P_{1}, \ldots, P_{r}\right\} \rightarrow \infty$.

Как известно, следствием оценки И. М. Виноградова явилась асимптотическая формула в проблеме Варинга в простых числах. В 2009 г. В. Н. Чубариков доказал, что последовательность $p^{n}$, где $p$ пробегает все простые числа, а $n-$ любое фиксированное натуральное число, является базисом конечного порядка для натурального ряда чисел.

\section{6. Бинарные аддитивные задачи с простыми числами}

В конце тридцатых годов прошлого столетия после решения И.М.Виноградовым тернарной проблемы Гольдбаха была открыта возможность оценки сверху мощности исключительного множества в бинарной проблеме Гольдбаха-Эйлера, т.е. оценки количества натуральных чисел $n$, не превосходящих $x \geqslant 6$, и не представимых суммой двух нечетных простых чисел. В 2002 г. Г. И. Архипов и В. Н. Чубариков оценили сверху $T(x)-$ количество $n \leqslant x$, не представимых в виде $\left[\lambda_{1} p_{1}\right]+\left[\lambda_{2} p_{2}\right]$, где $p_{1}, p_{2}-$ простые числа, $\lambda_{1}, \lambda_{2}-$ положительные вещественные числа, причем отношение $\lambda_{1} / \lambda_{2}$ является иррациональным алгебраическим числом. Имеет место неравенство

$$
T(x) \ll_{\varepsilon} x^{2 / 3+\varepsilon},
$$

где $\varepsilon>0$ - сколь угодно малое число и постоянная в знаке $\ll_{\varepsilon}-$ неэффективная. Первые более грубые результаты в этой задаче были получены ими в 1997 г. Заметим, что при $\lambda_{1}=\lambda_{2}=1$ удается получить только оценки вида

$$
T(x) \ll x^{1-\delta},
$$

где $\delta<1 / 10$.

\section{7. Метод И. М. Виноградова в теории оператора Бельтрами-Лапласа для случая трех переменных}

В трехмерном пространстве Лобачевского в модели Пуанкаре можно ввести координаты на верхнем полупространстве

$$
H^{3}=\left\{z=\left(x_{1}, x_{2}, y\right) \in \mathbf{R}^{3}: y>0\right\} .
$$

Как известно, функция

$$
u=u\left(z, z^{\prime}\right)=\frac{\left(x_{1}-x_{1}^{\prime}\right)^{2}+\left(x_{2}-x_{2}^{\prime}\right)^{2}+\left(y-y^{\prime}\right)^{2}}{y y^{\prime}}
$$

задает расстояние $d\left(z, z^{\prime}\right)$ между точками $z$ и $z^{\prime}$ по формуле

$$
u\left(z, z^{\prime}\right)=4 \operatorname{sh}^{2} \frac{d\left(z, z^{\prime}\right)}{2} .
$$

Шар с центром $z_{0}=\left(x_{10}, x_{20}, y_{0}\right)$ и радиусом $T$ в пространстве Лобачевского можно представить как шар в трехмерном евклидовом пространстве с центром в точке $\left(x_{10}, x_{20}, y_{0} \operatorname{ch} T\right)$ и радиусом $y_{0} \operatorname{sh} T$. 
Пусть $N\left(T, w_{0}, w\right)$ - количество элементов $g$ некоторой дискретной группы в пространстве Лобачевского, удовлетворяющих условию $d\left(w_{0}, g w\right) \leqslant T$.

Б. М. Левитан нашел асимптотическую формулу для $N\left(T, w_{0}, w\right)$ при $T \rightarrow \infty$, связанную с собственными значениями оператора Бельтрами-Лапласа.

Г. И. Архипов и В. Н. Чубариков использовали арифметический метод для вывода этой асимптотической формулы. Это дало им возможность получить нижнюю границу для первого собственного значения в рассматриваемой проблеме.

\section{8. Абсцисса и экспонента Карлсона в проблеме моментов дзета-функции Римана}

Абсциссой Карлсона называют величину $\sigma_{k}=\sigma(k)$, определяемую соотношениями

$$
\sigma_{k}=\inf \{M\},
$$

где $M-$ множество всех вещественных чисел $\sigma<1$, для которых справедлива оценка

$$
I_{k}=I_{k}(\sigma, T)=T^{-1} \int_{1}^{T}|\zeta(\sigma+i t)|^{2 k} d t \ll_{\varepsilon} T^{\varepsilon},
$$

где $k>0$ и $\varepsilon>0-$ произвольные вещественные числа.

Экспонентой Карлсона называют величину $m(\sigma)$, определяемую равенством

$$
m(\sigma)=2 f(\sigma)
$$

где $f(\sigma)$ - функция, обратная к $\sigma(k)$. Другими словами, функция $m(\sigma)$ определяется как $\sup \{m\}$, где $m>0$ таково, что при произвольном $\varepsilon>0$ выполняется оценка

$$
\int_{1}^{T}|\zeta(\sigma+i t)|^{2 m} d t \ll_{\varepsilon} T^{1+\varepsilon}
$$

Если справедлива гипотеза Линделёфа, утверждающая, что при $t \rightarrow \infty$ справедлива оценка

$$
\zeta(1 / 2+i t) \ll_{\varepsilon}|t|^{\varepsilon},
$$

то при всех $k>0$ справедливо равенство $\sigma_{k}=1 / 2$. Из оценки четвертого момента $\zeta(s)$ следует, что $\sigma_{k}=1 / 2$ при $0<k \leqslant 2$. При $k>2$ из стандартных соображений устанавливается, что $\sigma_{k}<1-1 / k$. В 1981 г. Д. Р. Хис-Браун доказал, что $\sigma_{8} \leqslant 5 / 8$, отсюда следует, что $m(5 / 8) \geqslant 8$. Более того, из оценки дзета-функции Римана в окрестности единичной прямой, впервые найденной Х.-Э. Рихертом (1960 г.), при 1/2<б<1 вытекает неравенство

$$
m(\sigma) \gg_{\varepsilon}(1-\sigma)^{-3 / 2} .
$$

Г. И. Архипов, Е. Е. Баядилов и В. Н. Чубариков (2003 г.) доказали следующее утверждение.

Пусть при некотором $а, 1 \leqslant a<20$, для всех $t \geqslant 1 u 1 / 2<\sigma<1$ справедлива оченка

$$
\zeta(\sigma+i t) \ll t^{a(1-\sigma)^{3 / 2}}
$$

u пусть $k_{0}=44-[22 / a]$. Тогда 
(а) для функции $\sigma_{k}$ при всех $k \geqslant 45$ имеет место оценка

$$
\sigma_{k} \leqslant 1-\frac{1}{\left(3 a\left(k-k_{0}\right)+\left(3 a\left(k-k_{0}\right)\right)^{1 / 2}\right)^{2 / 3}},
$$

(б) при всех $\sigma \geqslant \sigma_{1}=\frac{2701}{2880}$ справедливо неравенство

$$
\frac{m(\sigma)}{2} \geqslant k_{0}-1+\frac{1}{3 a(1-\sigma)^{3 / 2}}-\frac{1}{(3 a)^{1 / 2}(1-\sigma)^{3 / 4}} .
$$

\section{9. Аддитивная проблема Ингама}

Пусть $\tau(n)$ обозначает количество делителей натурального числа $n, k-$ натуральное число, и

$$
\sigma_{-1}(n)=\sum_{d \mid n} d^{-1}
$$

Тогда при $x \rightarrow \infty$ имеет место асимптотика

$$
I(x)=\sum_{n \leqslant x} \tau(n) \tau(n+k) \sim \frac{6}{\pi^{2}} \sigma_{-1}(k) x \ln ^{2} x,
$$

установленная А. Е. Ингамом в 1927 г. В 1931 г. Т. Эстерман нашел асимптотическую формулу

$$
I(x)=x\left(A_{0} \ln ^{2} x+A_{1} \ln x+A_{2}\right)+R(x),
$$

где

$$
R(x) \ll x^{11 / 12} \ln ^{17 / 3} x,
$$

$A_{0}, A_{1}, A_{2}$ - некоторые постоянные. В 1979 г. Д. И. Исмоилов, развивая элементарный метод Эстермана, получил новую оценку

$$
R(x) \ll x^{5 / 6+\varepsilon},
$$

где $\varepsilon>0$ - сколь угодно малая постоянная. В том же году ту же оценку, но равномерную по $k \leqslant x^{2 / 3}$ другим методом получил Д. Р. Хис-Браун.

В 2006 г. Г. И. Архипов и В. Н. Чубариков доказали, что

$$
R(x) \ll x^{3 / 4} \ln ^{4} x .
$$

\section{0. Вероятностная теория чисел}

Поясним, что особенность задач данного направления арифметики состоит в проявлении действия предельных законов теории вероятностей. Как подчеркивал А. Г. Постников, важнейшим инструментом в исследованиях по вероятностной теории чисел является метод моментов, открытый П. Л. Чебышёвым и А. А. Марковым. В работах А. Г. Постникова и М. П. Минеева разработан арифметический метод, позволяющий количественно оценивать скорости сходимости последовательностей к предельным распределениям.

Пусть $f(n)$ - периодическая функция натурального аргумента, имеющая период $p$. Тогда суммы вида $\sum_{n \leqslant h} f(n), \sum_{x<n \leqslant x+h} f(n)$ называются короткими, если длина интервала суммирования $h$ не превосходит величины $p$, и очень короткими, если значение $h$ является функцией от $p$, удовлетворяющей условиям $h \rightarrow \infty, \log h / \log p \rightarrow 0$ при $p \rightarrow \infty$. 


\section{1. Суммы символов Лежандра}

Далее, пусть $p-$ простое число и пусть

$$
\left(\frac{a}{p}\right)= \begin{cases}1, & \text { если } a \text { есть квадратичный вычет } \quad(\bmod p), \\ -1, & \text { если } a \text { есть квадратичный невычет } \quad(\bmod p), \\ 0, & \text { если } a \equiv 0 \quad(\bmod p),\end{cases}
$$

- символ Лежандра. Положим

$$
S_{h}(x)=\sum_{x<n \leqslant x+h}\left(\frac{n}{p}\right) .
$$

И. М. Виноградов и Д. Пойа (1918) при $1 \leqslant x<x+h \leqslant p$ доказали неравенство

$$
\left|S_{h}(x)\right|<\sqrt{p} \ln p .
$$

Д. А. Бёрджесс (1958) получил следующий результат.

Для произвольной постоянной $\varepsilon>0$ найдется функция $\delta(\varepsilon)>0$ такая, что

$$
S_{h}(x) \ll h p^{-\delta(\varepsilon)}
$$

при $h>p^{1 / 4+\varepsilon}$, т.е. количество квадратичных вычетов и невычетов по модулю $p$ в интервалах $[x, x+h]$ при $h>p^{1 / 4+\varepsilon}$, асимптотически одинаково.

Г. Давенпорт и П. Эрдёш (1952) для очень короткой суммы $S_{h}(x)$ символов Лежандра доказали следующее утверждение.

Пусть $M_{p}(\lambda)$ обозначает количество цельх чисел $x$ таких, что $0 \leqslant x<p u$ $S_{h}(x) \leqslant \lambda h^{1 / 2}$. Тогда при $p \rightarrow \infty$

$$
\frac{1}{p} M_{p}(\lambda) \rightarrow \frac{1}{\sqrt{2 \pi}} \int_{-\infty}^{\lambda} e^{-t^{2} / 2} d t
$$

для любого фиксированного $\lambda$.

\section{2. Суммы символов Лежандра по различным модулям}

Пусть $p_{1}, \ldots, p_{k}$ - различные простые числа, $Q=p_{1} \cdots p_{k}, 1 \leqslant x<x+h \leqslant Q, \varepsilon_{s}= \pm 1$ при $1 \leqslant s \leqslant k$, и пусть $T-$ количество целых чисел $n$, удовлетворяющих соотношениям

$$
\left(\frac{n+a_{1}}{p_{1}}\right)=\varepsilon_{1}, \quad \ldots, \quad\left(\frac{n+a_{k}}{p_{k}}\right)=\varepsilon_{k}
$$

для произвольных фиксированных целых чисел $a_{1}, \ldots, a_{k}$.

В. Н. Чубариков и его ученик Э. К. Жимбо (2001) получили следующие результаты.

Справедливо соотношение

$$
T=\frac{h}{2^{k}}+\theta \sqrt{Q} \ln Q,
$$

где $|\theta| \leqslant 1$ u $h>2^{k} \sqrt{Q} \ln Q$. 
Пусть

$$
S_{h}(x)=S_{h}\left(x ; p_{1}, \ldots, p_{k}\right)=\left(\frac{n+a_{1}}{p_{1}}\right) \cdots\left(\frac{n+a_{k}}{p_{k}}\right)
$$

- очень короткая сумма произведений символов Лежандра по различным модулям, т.е. $h \rightarrow \infty, \log h / \log Q \rightarrow 0$ при $Q \rightarrow \infty$. Пусть $N_{Q}\{x: \ldots\}$ обозначает количество целых чисел $x$, удовлетворяющих условиям, поставленным вместо точек. Справедливо следующее утверждение.

Пусть

$$
\xi=\xi(h, Q)=\frac{S_{h}(x)}{\sqrt{h}}
$$

- очень короткая нормированная сумма. Тогда при $Q \rightarrow \infty$

$$
\frac{1}{Q} N_{Q}\{x: \xi<y\} \rightarrow \frac{1}{\sqrt{2 \pi}} \int_{-\infty}^{y} e^{-y^{2} / 2} d y
$$

для любого фиксированного вещественного числа у.

\section{3. Очень короткая сумма по обратным к простым числам по произвольному простому модулю}

Пусть $p, q-$ простые числа. Пусть $q^{*}$ определяется сравнением $q q^{*} \equiv 1(\bmod p)$, $3<h<p, 1 \leqslant x \leqslant p-1$, и

$$
K_{h}(x)=\sum_{q \leqslant h} e^{2 \pi i x q^{*} / p} .
$$

В. Н. Чубариков и Э. К. Жимбо (2001) доказали следующую теорему.

$\Pi y c m b$

$$
\xi=\xi(x, h)=\left|\frac{K_{h}(x)}{\sqrt{h}}\right|^{2}
$$

- очень короткая нормированная сумма по обратным к простым по модулю р. Тогда при $p \rightarrow \infty$

$$
\frac{1}{p} N_{p}\{x: \xi<y\} \rightarrow 1-e^{-y}
$$

для любого фиксированного вещеественного числа у.

\section{4. Рациональные тригонометрические суммы по числам Фибоначчи}

Члены последовательности $\left\{f_{n}\right\}$, где $f_{0}=1, f_{1}=1$ и $f_{n+1}=f_{n}+f_{n-1}$ при $n \geqslant 1$, называются числами Фибоначчи. Пусть $m>1-$ натуральное число, $\lambda>0$ - постоянная, и пусть $N_{m}\{n: \ldots\}$ обозначает количество целых чисел $n$, удовлетворяющих условиям, которые указаны в скобках. Пусть, далее,

$$
S_{m}(h ; a)=\sum_{n=0}^{h-1} e^{2 \pi i a f_{n} / m}
$$


- очень короткая тригонометрическая сумма, и

$$
N_{m}(\lambda)=N_{m}\left\{a: 0 \leqslant a \leqslant m-1,\left|S_{m}(h ; a)\right|<\sqrt{\lambda h}\right\} .
$$

В. Н. Чубариков и его ученик Р. Н. Бояринов (2001) получили следующий результат.

Пусть $m \rightarrow \infty u h$ как функиия от $m$ удовлетворяет условиям $h=h(m) \rightarrow \infty u$ $h \leqslant \frac{1}{2} \log _{\tau} m$, где $\tau=\frac{1}{2}(\sqrt{5}+1)$. Тогда для любого фиксированного вещественного $\lambda>0$ справедливо соотношение

$$
\lim _{m \rightarrow \infty} \frac{N_{m}(\lambda)}{\lambda}=1-e^{-\lambda}
$$

\section{1. $L$-функции Дирихле по модулю, равному степени простого числа}

В 1955 г. А. Г. Постников сделал выдающееся открытие в p-адическом анализе. Оно позволило выразить сумму значений характеров Дирихле по примарному модулю через рациональную тригонометрическую сумму. Им была получена принципиально новая оценка сумм неглавных характеров Дирихле по модулю, равному степени нечетного простого числа, и установлены более точные границы нулей соответствующих $L$-функций Дирихле, чем имеющиеся границы для произвольного модуля. Эта работа была продолжена С. М. Розиным, А. А. Карацубой, Н. Г. Чудаковым, В. Н. Чубариковым и др. В 2000 г. Владимир Николаевич и его ученик Б. А. Турешбаев в окрестности единичной прямой получили следующие оценки.

Пусть $\chi-$ примитивный характер по модулю $D=p^{k}, p-$ нечетное простое число, $1-4 \gamma<\sigma<1$,

$$
b=\frac{2}{3 \sqrt{3 \gamma}}
$$

A> 0 и 1/1024 > $\gamma>0-$ некоторые постоянные. Тогда

(a1) $n$ ри $p \leqslant e^{A k^{2}},|t| \leqslant 2 D$

$$
|L(s, \chi)| \ll \frac{D^{b(1-\sigma)^{3 / 2}} \ln ^{2 / 3} D}{(1-\sigma)^{1 / 4} \ln ^{1 / 6} D+1},
$$

(a2) при $D \geqslant D_{0}=D_{0}(A)$ функция $L(s, \chi)$ не имеет нулей в области

$$
|t| \leqslant D, \quad \sigma \geqslant 1-\frac{c}{\ln ^{2 / 3} D(\ln \ln D)^{1 / 3}}, \quad c=\frac{1}{500 b^{2 / 3}},
$$

(б1) при $p \leqslant e^{A \ln ^{2 / 3}|t|},|t| \geqslant D$

$$
|L(s, \chi)| \ll \frac{|t|^{b(1-\sigma)^{3 / 2}} \ln ^{2 / 3}|t|}{(1-\sigma)^{1 / 4} \ln ^{1 / 6}|t|+1},
$$

(б2) при $D \geqslant D_{0}=D_{0}(A)$ функичия $L(s, \chi)$ не имеет нулей в области

$$
|t| \geqslant D, \quad \sigma \geqslant 1-\frac{c}{\ln ^{2 / 3}|t|(\ln \ln |t|)^{1 / 3}} .
$$




\section{2. Математический анализ}

Современный взгляд на математический анализ как на научную дисциплину подразумевает включение в его состав теории функций действительного и комплексного переменных, теорию специальных функций и функционального анализа. С этой точки зрения исследования В. Н. Чубарикова по теории тригонометрических интегралов и спектральной теории оператора Лапласа-Бельтрами можно в равной степени рассматривать в рамках и математического анализа, и теории чисел.

С другой стороны, изложение математического анализа в вузовской программе до сих пор оставляет достаточное количество методологических и научных проблем. От В. Н. Чубарикова, как соавтора учебника по университетскому курсу математического анализа, потребовалось усовершенствования подхода к понятию предела: введения понятий фундаментальной и монотонной последовательностей по базам множеств, доказательства эквивалентности пределов функций по Коши и по Гейне, вывода равенства верхнего предела функции и верхнего предельного числа по базам множеств, а также вывода равенства двойных и повторных пределов по базам множеств; упрощения применения формул Эйлера, Абеля и Пуассона суммирования значений функций по целым точкам, вычисления значений сумм Гаусса, нового подхода к построению теории поверхностных интегралов, что позволило дать простое доказательство общей формулы Стокса для кусочно-гладкой ориентированной поверхности произвольной размерности в многомерном пространстве.

\section{3. Арифметические задачи криптографии}

Совместно с М. П. Минеевым, В. Н. Чубариков предложил новый арифметический подход к задаче об искажении знаков в шифрах простой замены и Виженера. Для этой цели они использовали операции извлечения корня квадратного и возведения в квадрат по модулю простого числа. Ими найдено также применение китайской теоремы об остатках для арифметического варианта шифра Виженера. Им удалось построить цифровую подпись на основе $p$-адических чисел. В 2011 г. они получили новый блочный шифр, используя теорию разбиений натурального числа и решение системы нелинейных сравнений по специальным модулям.

\section{4. История математики}

В. Н. Чубариков постоянно проявляет интерес к деятельности математиков прошлого и настоящего. Свое осмысление их творчества он многократно представлял в своих докладах и публикациях. Он прослеживал преемственность в работах Эйлера, Чебышёва, Маркова, Виноградова. В частности, он отметил, что знаменитые исследования Римана восходят к работам Чебышёва. Исследуя работы И. М. Виноградова, В. Н. Чубариков явно выделил его изобретения в технике, на основе которых были сделаны им выдающиеся математические открытия, ставшие сейчас основой стандартного аппарата аналитической теории чисел. Анализируя научное наследие Н. Г. Чудакова, он перечислил основные достижения мировой математики в области аналитической теории чисел и высказал мнение, что алгебраические построения в ней не вполне отражают арифметическую природу ее классических задач.

В 2001 г. на 4-й Международной конференции «Современные проблемы теории чисел и ее приложения» В. Н. Чубариков дал комментарии к письму от 16 апреля 1946 г. 
Н. Н. Лузина И. М. Виноградову, посвященного арифметическим результатам П. Ферма относительно метода доказательства «последнего предложения» и простоты чисел Ферма. Начиная с девяностых годов прошлого века, В. Н. Чубариков неоднократно выступал на конференциях, на семинарах механико-математического факультета МГУ с докладами, посвященными 100-летию Н. Г. Чудакова, 180 и 190-летию П. Л. Чебышёва, 110 и 120летию И. М. Виноградова, 100-летию К. К. Марджанишвили, к 300-летию Л. Эйлера и 300-летию М. В. Ломоносова, 60-летию А. А. Карацубы и 60-летию Г. И. Архипова. В. Н. Чубариков стал редактором и одним из инициаторов издания «Избранных трудов» А. Г. Постникова.

\section{5. Популяризация математики}

Будучи аспирантом, В. Н. Чубариков в 1976 г. начал преподавать математику в колмогоровской школе-интернате при МГУ. Часть материала этой работы содержится в книгах $[4,5,6]$. В последние годы он регулярно выступает с популярными лекциями по математике на различных факультетах МГУ и в других вузах страны.

В. Н. Чубариков - выдающийся профессор механико-математического факультета МГУ. С 1989 г. он читает основной курс математического анализа для студентов-математиков механико-математического факультета МГУ. Его лекции отличает тщательная проработка материала и представление нового взгляда на, казалось бы, давно решенные проблемы. Свои лекции, как соавтор известного учебника, он обобщил в книге [4]. Работа его специальных курсов и специальных семинаров отражена в учебных пособиях [5, 6, 7].

В. Н. Чубариков подготовил 27 кандидатов наук и 3 доктора наук. Дипломные работы под его руководством выполнили несколько сотен выпускников механико-математического факультета МГУ. Он является соавтором трех научных монографий $[1,2,3]$.

Много сил В. Н. Чубариков отдает научно-организационной работе. В течение ряда он возглавляет Ученый совет механико-математического факультета МГУ, докторский совет по математике по защитам диссертаций при МГУ. Он является главным редактором научных журналов «Вестник МГУ. Серия І. Математика. Механика», «Чебышёвский сборник», входит в состав редакционных коллегий журналов «Дискретная математика», «Вопросы атомной физики. Математическое моделирование физических процессов» и «Евразийский математический журнал» (издающийся в Казахстане). В. Н. Чубариков был переводчиком и редактором перевода на русский язык книг К. Хооли, П. Рибенбойма, Р. Крэндалла и К. Померанца.

В. Н. Чубариков встречает свое шестидесятилетие в расцвете творческих сил. В своих работах он разрешает трудные арифметические вопросы, проявляя комбинаторную изобретательность в сочетании с красивыми приложениями аналитических и арифметических методов исследования.

\section{Список литературы}

1. Архипов Г. И., Карацуба А. А., Чубариков В. Н., Кратные тригонометрические суммы. Труды Математического института имени В. А. Стеклова АН СССР (1980) 151, 3-128.

2. Архипов Г. И., Карацуба А. А., Чубариков В. Н., Теория кратных тригонометрических сумм. Наука, Москва, 1987.

3. Arkhipov G. I., Chubarikov V. N., Karatsuba A. A., Trigonometric sums in number theory and analysis. de Gruyter, Berlin, 2004. 
4. Архипов Г. И., Садовничий В. А., Чубариков В. Н., Лекции по математическому анализу. Дрофа, Москва, 2006.

5. Гашков С. Б., Чубариков В. Н., Арифметика. Алгоритмы. Сложность вычислений. Дрофа, Москва, 2004.

6. Чубариков В. Н., Элементы арифметики. Изд-во мех.-мат. ф-та МГУ, Москва, 2007.

7. Минеев М. П., Чубариков В. Н., Лекиии по арифметическим вопросам криптографии. Изд-во мех.-мат. ф-та МГУ, Москва, 2010.

Статья поступила 6.08.2011. 